\title{
Function Synthesis Algorithm of RTD-Based Universal Threshold Logic Gate
}

\author{
Maoqun Yao, ${ }^{1}$ Kai Yang, ${ }^{1}$ Congyuan $\mathrm{Xu},{ }^{2}$ and Jizhong Shen ${ }^{2}$ \\ ${ }^{1}$ Hangzhou Institute of Service Engineering, Hangzhou Normal University, Hangzhou 311121, China \\ ${ }^{2}$ Department of Information Science \& Electronic Engineering, Zhejiang University, Hangzhou 310027, China \\ Correspondence should be addressed to Maoqun Yao; yaomaoqun@163.com
}

Received 19 March 2015; Revised 13 May 2015; Accepted 31 May 2015

Academic Editor: Georgios Sirakoulis

Copyright (c) 2015 Maoqun Yao et al. This is an open access article distributed under the Creative Commons Attribution License, which permits unrestricted use, distribution, and reproduction in any medium, provided the original work is properly cited.

\begin{abstract}
The resonant tunneling device (RTD) has attracted much attention because of its unique negative differential resistance characteristic and its functional versatility and is more suitable for implementing the threshold logic gate. The universal logic gate has become an important unit circuit of digital circuit design because of its powerful logic function, while the threshold logic gate is a suitable unit to design the universal logic gate, but the function synthesis algorithm for the $n$-variable logical function implemented by the RTD-based universal logic gate (UTLG) is relatively deficient. In this paper, three-variable threshold functions are divided into four categories; based on the Reed-Muller expansion, two categories of these are analyzed, and a new decomposition algorithm of the three-variable nonthreshold functions is proposed. The proposed algorithm is simple and the decomposition results can be obtained by looking up the decomposition table. Then, based on the Reed-Muller algebraic system, the arbitrary $n$-variable function can be decomposed into three-variable functions, and a function synthesis algorithm for the $n$-variable logical function implemented by UTLG and XOR2 is proposed, which is a simple programmable implementation.
\end{abstract}

\section{Introduction}

With the improvement in integrated circuit integration, the complementary metal oxide semiconductor (CMOS) technology is gradually approaching its physical limitations. The resonant tunneling device (RTD) has better performance and features, such as negative differential resistance characteristic, self-latching, high speed, and functional versatility $[1,2]$. The universal logic gate, which has a powerful logic function, has become an important unit to implement $n$-variable logical functions [3], and the RTD is more suitable for implementing the universal logic gate because of its negative differential resistance characteristic [4-6]. So, the RTD will probably become the main electronic device in the next generation of integrated circuits $[7,8]$.

Though the circuit of an $n$-variable logical function implemented by the universal logic gate will be simpler, a different universal logic gate requires its corresponding synthesis algorithm to implement a function. Some function synthesis algorithms have been proposed in the literature [912], but these algorithms are not suitable for implementing an arbitrary $n$-variable function by the RTD-based universal threshold logic gate (UTLG) [13]. And the algorithm [14] which can implement a three-variable nonthreshold function by UTLGs is relatively complicated, and the implemented circuit structure is also complicated.

In this paper, based on the Reed-Muller expansion, the three-variable nonthreshold functions are classified. Two categories of these are analyzed, and a new decomposition algorithm of the three-variable nonthreshold functions is proposed. Then a function synthesis algorithm which can implement an arbitrary $n$-variable logical function by UTLGs is proposed. The proposed function synthesis algorithm provides a new scheme for designing integrated circuits by RTD devices.

\section{Background}

2.1. Threshold Logic. A threshold logic gate is defined as a logic gate with $n$ binary input variables and a single binary output. Its internal parameters are as follows: $n$ binary input 
variables, $\left\{x_{i}\right\}(i=1,2, \ldots, n)$, a set of integer weights, $\left\{w_{i}\right\}(i=1,2, \ldots, n)$, and a threshold $T$ and an output $f$, such that its input-output relationship can be expressed as [15]

$$
f= \begin{cases}1, & \text { if } \sum_{i=1}^{n} w_{i} x_{i} \geq T \\ 0, & \text { otherwise. }\end{cases}
$$

Formula (1) can also be presented as $f=\left\langle w_{1} x_{1}+\right.$ $\left.\cdots+w_{n} x_{n}\right\rangle_{T}$. If a logic function can be implemented with a single threshold logic gate, the function is called a threshold function; otherwise, it is called a nonthreshold function [15].

2.2. Spectral Technique. Spectral technique is a mathematical transformation method. It can convert binary data from the Boolean domain into the spectral domain by matrix transformations, and the information will not be lost [16]. In the spectral domain $\{-1,+1\}$, for an $n$-variable logical function $f\left(x_{1}, \ldots, x_{n}\right)$, its input and output have $2^{n}$ kinds of states, and the truth vector is

$$
\begin{aligned}
Y= & (f(+1,+1, \ldots,+1) \\
& \cdot f(+1,+1, \ldots,-1) \cdots f(-1,-1, \ldots,-1))^{T} .
\end{aligned}
$$

The spectral-coefficient vector $R$ is given by

$$
R=\left(\begin{array}{lllllll}
r_{0} & r_{1} & \cdots & r_{n} & r_{12} & \cdots & r_{1, \ldots, n}
\end{array}\right)^{T}=T^{n} \cdot Y,
$$

where $T^{n}$ is a $2^{n} \times n$ Rademacher-Walsh matrix.

As for a three-variable function, the spectral-coefficient vector is $R=\left(\begin{array}{llllllll}r_{0} & r_{3} & r_{2} & r_{23} & r_{1} & r_{13} & r_{12} & r_{123}\end{array}\right)^{T}$, where $r_{0}$ is a zero-order spectral-coefficient, and $r_{i}, r_{i j}$, and $r_{i j k}$ are one-order, two-order, and three-order spectral coefficients, respectively.

2.3. Reed-Muller Expansion. A Reed-Muller expansion is a standard expansion in the AND/XOR algebraic system. A given function can be expressed as the XOR of basic entry; its coefficient is called the RM expansion coefficient [17]. Given an $n$-variable function $f\left(x_{1}, \ldots, x_{n}\right)$, its RM expansion coefficient vector $B$ ( 0 -polarity; in this paper we only use 0 polarity expansion coefficient) is

$$
B=[T]^{\otimes n} \cdot F,
$$

where $[T]^{\otimes n}=\underbrace{[T] \otimes[T] \otimes \cdots \otimes[T]}_{n},[T]=\left(\begin{array}{ll}1 & 0 \\ 1 & 1\end{array}\right), \otimes$ is the Kronecker product, and $F$ is the truth vector of the function in the Boolean domain. As for a three-variable function $f\left(x_{1}, x_{2}, x_{3}\right)$, its RM expansion is

$$
\begin{array}{r}
f\left(x_{1}, x_{2}, x_{3}\right)=b_{0} \oplus b_{1} x_{3} \oplus b_{2} x_{2} \oplus b_{3} x_{2} x_{3} \oplus b_{4} x_{1} \\
\\
\oplus b_{5} x_{1} x_{3} \oplus b_{6} x_{1} x_{2} \oplus b_{7} x_{1} x_{2} x_{3} .
\end{array}
$$

\section{Decomposition Algorithm of Three-Variable Nonthreshold Functions}

In this section, the three-variable nonthreshold functions are classified, and a new decomposition algorithm of the threevariable nonthreshold functions is proposed.
TABLE 1: Spectral-coefficient $\left|r_{i}\right|$ classification of all the threevariable threshold functions.

\begin{tabular}{lllllllll}
\hline$n$ & \multicolumn{3}{c}{$\left|r_{i}\right|$} & & \multicolumn{5}{c}{$\left|a_{i}\right|$} \\
\hline \multirow{3}{n}{$n=3$} & 8 & 0 & 0 & 0 & 1 & 0 & 0 & 0 \\
& 6 & 2 & 2 & 2 & 2 & 1 & 1 & 1 \\
& 4 & 4 & 4 & 0 & 1 & 1 & 1 & 0 \\
\hline
\end{tabular}

3.1. Determine the Three-Variable Threshold Function. The zero-order and one-order spectral-coefficient can determine whether a function is a threshold function [16]. The spectralcoefficient classification table of all the three-variable threshold functions is given in Table 1, and some conclusions as follows [16].

(1) The zero-order and one-order spectral-coefficients of the given function are calculated and arranged in numerically descending magnitude order, and this is the $\left|r_{i}\right|$ of the function; if the $\left|r_{i}\right|$ appear in Table 1, the function is a threshold function. The threshold value of a threshold function and weights can be obtained by $\left|a_{i}\right|$ in Table 1 . The relationship between the $\left|w_{i}\right|$ and listed $\left|a_{i}\right|$ value is

$$
\left|w_{i}\right|=\left|a_{i}\right| \quad i=1,2,3
$$

The sign of $w_{i}$ is the same as $r_{i}$. The threshold $T$ can be calculated as

$$
T=\frac{1}{2}\left(\sum_{i=0}^{3} a_{i}+1\right)
$$

(2) If the function is not a threshold function, the derived number $\left|r_{i}\right|$ will not appear in Table 1.

3.2. New Decomposition Algorithm of Three-Variable Nonthreshold Functions. According to the properties of the spectral coefficient, the three-variable nonthreshold function can be decomposed into three-variable threshold functions by a series of conversions [14], but for some three-variable nonthreshold functions, this algorithm [14] is relatively complex. So, we will propose a simpler algorithm which can decompose those three-variable nonthreshold functions into three-variable threshold functions.

For all the three-variable functions, there are 104 threshold functions and 152 nonthreshold functions [16].

First, according to Table 1, the 104 threshold functions are divided into three categories [16].

(1) Among the absolute values of all the zero-order and one-order spectral-coefficients, there is one number equal to 8 and three numbers equal to 0 .

(2) Among the absolute values of all the zero- and oneorder spectral-coefficients, there is one number equal to 6 and three numbers equal to 2 .

(3) Among the absolute values of all the zero-order and one-order spectral-coefficients, there is one number equal to 0 and three numbers equal to 4 . 
Then, the 152 nonthreshold functions can be divided into four categories.

(1) Among the absolute values of all the spectral coefficients, the maximum number is from the two-order spectral coefficient.

(2) Among the absolute values of all the spectral coefficients, the maximum number is from the three-order spectral coefficient.

(3) Among the absolute values of all the zero-order and one-order spectral-coefficients, there is one number equal to 4 .

(4) Among the absolute values of all the zero-order and one-order spectral-coefficients, there are two numbers equal to 4 .

The numbers of these four categories of three-variable nonthreshold functions are $54,18,33$, and 47 , respectively. If the function belongs to the second and third categories of the three-variable nonthreshold functions, the algorithm [14] which decomposes the function will be relatively complex. A nonthreshold function is decomposed into the threshold function by the conversion of $f(x) \oplus x_{i}$ and $x_{i} \leftrightarrow x_{i} \oplus x_{j}$, so we propose a new decomposition algorithm of the three-variable nonthreshold functions based on the Reed-Muller algebraic system, and the decomposition process is as follows.

(1) The RM expansion coefficient vectors of all the three-variable threshold functions are calculated as a reference table.

(2) The RM expansion coefficient vector of the given function is calculated.

(3) Search the reference table and find two threshold functions in which the XOR result of their RM expansion coefficient vectors just equals the calculated RM expansion coefficient vector in step 2.

(4) The three-variable nonthreshold function can be expressed as the XOR of the found threshold functions of step 3.

After applying the process to the second and third categories of the three-variable nonthreshold functions, except two special functions, $f=x_{1} \oplus x_{2} \oplus x_{3}$ and $f=$ $\overline{x_{1} \oplus x_{2} \oplus x_{3}}$, the others can be presented by the XOR of two threshold functions; that is, $f\left(x_{1}, x_{2}, x_{3}\right)=f_{1}\left(x_{1}, x_{2}, x_{3}\right) \oplus$ $f_{2}\left(x_{1}, x_{2}, x_{3}\right)$, where $f_{1}$ and $f_{2}$ are threshold functions. The decomposition results of the second and third categories of the three-variable nonthreshold functions are shown in Tables 2 and 3. In the tables, each vector of the columns is the output of the three-variable function for an 8-input combination from 000 to 111 . Taking the first column of the first vector $\left(\begin{array}{llllllll}0 & 0 & 0 & 1 & 0 & 1 & 1 & 0\end{array}\right)$ as an example in Table 2, it expresses the three-variable function $f=\sum m_{i}(i=3,4)$, where $m_{i}$ is the minterm of the function, and $f$ can be expressed as $f=f_{1} \oplus f_{2}$, where $f_{1}=\sum m_{i}(i=3,4,5,6,7)$ and $f_{2}=\sum m_{i}(i=5,6,7)$, and they are the three-variable threshold functions denoted by the second and third columns of the first row in Table 2.

\section{The Synthesis Algorithm of $n$-Variable Function Based on UTLG}

The literature [13] proposed an RTD-based universal logic gate (UTLG) which can implement an arbitrary threevariable threshold function with a single UTLG. Figure 1 shows the schematic and symbol of UTLG; its input-output relationship can be expressed as

$$
f= \begin{cases}1, & 2 c_{1}+c_{2}+c_{3}-2 c_{4}-c_{5}-c_{6} \geq 1 \\ 0, & \text { otherwise. }\end{cases}
$$

The first and fourth categories of the three-variable nonthreshold functions can be implemented by 3 UTLGs, and the second and third categories of the three-variable nonthreshold functions can be implemented by 7 UTLGs [13]. In our proposed decomposition algorithm of three-variable nonthreshold functions we introduced a bivariate XOR function, which cannot be implemented by a single UTLG, so according to the structure of MOBILE circuit [18], we design an RTD-based bivariate XOR gate (XOR2). Figure 2 shows the schematic and symbol of XOR2. Thus, the second and third categories of the three-variable nonthreshold functions can be implemented by 2 UTLGs and 1 XOR2, and it simplifies the circuit. Figure 3 shows the simulation results of XOR2 circuit by HSPICE; the parameters of RTD and HFET are the same as UTLG circuit [13], and as observed from Figure 3, the proposed XOR2 has the correct logic functionality.

Currently, there is no algorithm which can implement arbitrary $n$-variable functions by the UTLG. Therefore, we proposed an $n$-variable function synthesis algorithm based on UTLG.

First, we analyze the RM expansion of a four-variable function $f\left(x_{1}, x_{2}, x_{3}, x_{4}\right)[17] ; f=b_{0} \oplus b_{1} x_{4} \oplus b_{2} x_{3} \oplus b_{3} x_{3} x_{4} \oplus$ $b_{4} x_{2} \oplus b_{5} x_{2} x_{4} \oplus b_{6} x_{2} x_{3} \oplus b_{7} x_{2} x_{3} x_{4} \oplus b_{8} x_{1} \oplus b_{9} x_{1} x_{4} \oplus b_{10} x_{1} x_{3} \oplus$ $b_{11} x_{1} x_{3} x_{4} \oplus b_{12} x_{1} x_{2} \oplus b_{13} x_{1} x_{2} x_{4} \oplus b_{14} x_{1} x_{2} x_{3} \oplus b_{15} x_{1} x_{2} x_{3} x_{4}$.

By observation, the last eight terms of the RM expansion are equal to the first eight terms of the RM expansion by multiplying $x_{1}$, respectively, and the operations of terms in the RM expansion function are XOR, so the four-variable function can be expressed as $f=f_{11} \oplus x_{1} f_{12}$, the variables of $f_{11}$ are $x_{2}, x_{3}$ and $x_{4}$, and the RM expansion coefficient vector of $f_{11}$ is equal to the first eight elements of the RM expansion coefficient vector of $f$. The variables of $f_{12}$ are also $x_{2}, x_{3}$, and $x_{4}$, and the RM expansion coefficient vector of $f_{12}$ is equal to the last eight elements of the RM expansion coefficient vector of $f$.

For an $n$-variable function, based on the Reed-Muller algebraic system [17], it can also be expressed as $f=f_{11} \oplus$ $x_{1} f_{12}$, so we propose the synthesis algorithm of $n$-variable functions based on UTLG; its process is as follows.

(1) Calculate the RM expansion coefficient vector $B$ of the given $n$-variable function $f$.

(2) In the first decomposition, $f=f_{11} \oplus x_{1} f_{12}$, the subfunction $f_{11}$ is the $n-1$ variable of the function except $x_{1}$, and its RM expansion coefficient vector $B_{1}$ is equal to the first $n / 2$ elements of $B$; the subfunction 
TABLE 2: The second category of the three-variable nonthreshold functions.

\begin{tabular}{|c|c|c|c|c|c|}
\hline$f=\sum m_{i}$ & $f_{1}=\sum m_{i}$ & $f_{2}=\sum m_{i}$ & $f=\sum m_{i}$ & $f_{1}=\sum m_{i}$ & $f_{2}=\sum m_{i}$ \\
\hline 01234567 & 01234567 & 01234567 & 01234567 & 01234567 & 01234567 \\
\hline $\begin{array}{lllllllll}0 & 0 & 0 & 1 & 0 & 1 & 1 & 0\end{array}$ & $\begin{array}{lllllllll}0 & 0 & 0 & 1 & 0 & 0 & 0 & 1\end{array}$ & $\begin{array}{lllllllll}0 & 0 & 0 & 0 & 0 & 1 & 1 & 1\end{array}$ & 1000001100 & $\begin{array}{lllllllll}0 & 0 & 0 & 0 & 1 & 0 & 0 & 0\end{array}$ & 100000111000 \\
\hline $\begin{array}{llllllll}0 & 0 & 1 & 0 & 1 & 0 & 0 & 1\end{array}$ & $\begin{array}{llllllll}0 & 0 & 1 & 0 & 1 & 0 & 1 & 0\end{array}$ & $\begin{array}{llllllll}0 & 0 & 0 & 0 & 0 & 0 & 1 & 1\end{array}$ & 10010010 & $\begin{array}{lllllllll}0 & 0 & 1 & 0 & 0 & 0 & 0 & 0\end{array}$ & 101100010 \\
\hline $\begin{array}{llllllll}0 & 1 & 0 & 0 & 1 & 0 & 0 & 1\end{array}$ & $\begin{array}{lllllllll}0 & 1 & 0 & 0 & 0 & 1 & 0 & 0\end{array}$ & $\begin{array}{llllllll}0 & 0 & 0 & 0 & 1 & 1 & 0 & 1\end{array}$ & 1001101000 & $\begin{array}{lllllllll}0 & 0 & 1 & 0 & 1 & 0 & 1 & 1\end{array}$ & 10011111111 \\
\hline $\begin{array}{llllllll}0 & 1 & 1 & 0 & 0 & 0 & 0 & 1\end{array}$ & $\begin{array}{llllllll}0 & 0 & 0 & 1 & 0 & 0 & 0 & 1\end{array}$ & $\begin{array}{lllllllllll}0 & 1 & 1 & 1 & 0 & 0 & 0 & 0\end{array}$ & $\begin{array}{lllllll}10 & 0 & 1 & 0 & 1 & 1 & 1\end{array}$ & $\begin{array}{lllllllll}0 & 0 & 0 & 1 & 0 & 1 & 1 & 1\end{array}$ & 100000000000 \\
\hline $\begin{array}{lllllllll}0 & 1 & 1 & 0 & 1 & 0 & 0 & 0\end{array}$ & $\begin{array}{lllllllll}0 & 0 & 0 & 1 & 0 & 1 & 1 & 1\end{array}$ & $0 \begin{array}{llllllll}0 & 1 & 1 & 1 & 1 & 1 & 1 & 1\end{array}$ & 10011110 & $\begin{array}{llllllll}0 & 0 & 0 & 1 & 0 & 0 & 0 & 1\end{array}$ & $\begin{array}{lllllllll}1 & 0 & 0 & 0 & 1 & 1 & 1 & 1\end{array}$ \\
\hline $\begin{array}{lllllllll}0 & 1 & 1 & 0 & 1 & 0 & 1 & 1\end{array}$ & $\begin{array}{llllllll}0 & 1 & 0 & 0 & 0 & 1 & 0 & 0\end{array}$ & $\begin{array}{lllllllll}0 & 0 & 1 & 0 & 1 & 1 & 1 & 1\end{array}$ & 101110110 & $\begin{array}{llllllll}0 & 0 & 0 & 0 & 0 & 1 & 0 & 1\end{array}$ & $\begin{array}{llllllll}1 & 0 & 1 & 1 & 0 & 0 & 1 & 1\end{array}$ \\
\hline $\begin{array}{llllllll}0 & 1 & 1 & 0 & 1 & 1 & 0 & 1\end{array}$ & $\begin{array}{lllllllll}0 & 0 & 1 & 0 & 0 & 0 & 0 & 0\end{array}$ & $\begin{array}{llllllll}0 & 1 & 0 & 0 & 1 & 1 & 0 & 1\end{array}$ & $\begin{array}{llllllll}11 & 0 & 1 & 0 & 1 & 1 & 0\end{array}$ & $\begin{array}{lllllllll}0 & 0 & 0 & 0 & 0 & 0 & 1 & 1\end{array}$ & $\begin{array}{lllllllll}1 & 1 & 0 & 1 & 0 & 1 & 0 & 1\end{array}$ \\
\hline $\begin{array}{lllllllll}0 & 1 & 1 & 1 & 1 & 0 & 0 & 1\end{array}$ & 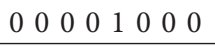 & $\begin{array}{lllllllll}0 & 1 & 1 & 1 & 0 & 0 & 0 & 1\end{array}$ & $\begin{array}{llllllll}1 & 1 & 1 & 0 & 1 & 0 & 0 & 1\end{array}$ & $\begin{array}{llllllll}0 & 0 & 0 & 0 & 0 & 0 & 0 & 1\end{array}$ & $\begin{array}{llllllll}1 & 1 & 1 & 0 & 1 & 0 & 0 & 0 \\
\end{array}$ \\
\hline
\end{tabular}

TABLE 3: The third category of the three-variable nonthreshold functions.

\begin{tabular}{|c|c|c|c|c|c|}
\hline$f=\sum m_{i}$ & $f_{1}=\sum m_{i}$ & $f_{2}=\sum m_{i}$ & $f=\sum m_{i}$ & $f_{1}=\sum m_{i}$ & $f_{2}=\sum m_{i}$ \\
\hline 012334567 & 01234567 & 01234567 & 01234567 & 01234567 & 01234567 \\
\hline $\begin{array}{llllllll}0 & 0 & 0 & 1 & 1 & 0 & 0 & 0\end{array}$ & $\begin{array}{lllllllll}0 & 0 & 0 & 1 & 1 & 1 & 1 & 1\end{array}$ & $\begin{array}{lllllllll}0 & 0 & 0 & 0 & 0 & 1 & 1 & 1\end{array}$ & $\begin{array}{lllllllll}1 & 0 & 0 & 0 & 0 & 1 & 0 & 0\end{array}$ & $\begin{array}{lllllllll}1 & 0 & 0 & 0 & 0 & 0 & 0 & 0\end{array}$ & $\begin{array}{lllllllll}0 & 0 & 0 & 0 & 0 & 1 & 0 & 0\end{array}$ \\
\hline $\begin{array}{lllllllll}0 & 0 & 0 & 1 & 1 & 1 & 1 & 0\end{array}$ & $\begin{array}{lllllllll}0 & 0 & 1 & 0 & 0 & 0 & 0 & 0\end{array}$ & $\begin{array}{llllllll}0 & 0 & 0 & 0 & 0 & 0 & 0 & 1\end{array}$ & 10000111 & 10000000 & $\begin{array}{lllllllll}0 & 0 & 0 & 0 & 0 & 1 & 1 & 1\end{array}$ \\
\hline $\begin{array}{lllllllll}0 & 0 & 1 & 0 & 0 & 1 & 0 & 0\end{array}$ & $\begin{array}{llllllll}0 & 0 & 1 & 0 & 0 & 0 & 1 & 1\end{array}$ & $\begin{array}{llllllll}0 & 0 & 0 & 0 & 0 & 1 & 1 & 1\end{array}$ & 1001010011 & 10100000 & $\begin{array}{llllllll}0 & 0 & 1 & 1 & 0 & 0 & 1 & 1\end{array}$ \\
\hline $\begin{array}{lllllllll}0 & 0 & 1 & 0 & 1 & 1 & 0 & 1\end{array}$ & $\begin{array}{llllllll}0 & 0 & 1 & 0 & 1 & 1 & 1 & 1\end{array}$ & $\begin{array}{lllllllll}0 & 0 & 0 & 0 & 0 & 0 & 1 & 0\end{array}$ & $\begin{array}{lllllll}10 & 0 & 1 & 0 & 1 & 0 & 1\end{array}$ & 1000101010 & $\begin{array}{lllllllll}0 & 0 & 0 & 1 & 1 & 1 & 1 & 1\end{array}$ \\
\hline $\begin{array}{lllllllll}0 & 0 & 1 & 1 & 0 & 1 & 1 & 0\end{array}$ & $\begin{array}{llllllll}0 & 0 & 1 & 1 & 0 & 1 & 1 & 1\end{array}$ & $\begin{array}{lllllllll}0 & 0 & 0 & 0 & 0 & 0 & 0 & 1\end{array}$ & 100111010 & 1010101010 & $\begin{array}{lllllllll}0 & 0 & 1 & 1 & 0 & 0 & 0 & 0\end{array}$ \\
\hline $\begin{array}{lllllllll}0 & 0 & 1 & 1 & 1 & 0 & 0 & 1\end{array}$ & $\begin{array}{llllllllll}0 & 0 & 1 & 1 & 1 & 0 & 1 & 1\end{array}$ & $\begin{array}{lllllllll}0 & 0 & 0 & 0 & 0 & 0 & 1 & 0 \\
0 & 0 & 0 & 0 & 0 & 1\end{array}$ & 10011100 & $\begin{array}{lllllllll}0 & 0 & 1 & 1 & 0 & 0 & 1 & 1\end{array}$ & $\begin{array}{lllllll}10 & 1 & 0 & 1 & 1 & 1 & 1\end{array}$ \\
\hline $\begin{array}{lllllllll}0 & 1 & 0 & 0 & 0 & 0 & 1 & 0\end{array}$ & $\begin{array}{lllllllll}0 & 1 & 0 & 0 & 0 & 1 & 0 & 1\end{array}$ & $\begin{array}{lllllllll}0 & 0 & 0 & 0 & 0 & 1 & 1 & 1\end{array}$ & 1010100110 & 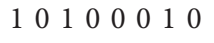 & $\begin{array}{lllllllll}0 & 0 & 0 & 0 & 0 & 1 & 0 & 0\end{array}$ \\
\hline $\begin{array}{lllllllll}0 & 1 & 0 & 0 & 1 & 0 & 1 & 0 \\
0 & 1 & 0 & 1 & 0 & & 1 & 0\end{array}$ & $\begin{array}{lllllllll}0 & 1 & 0 & 0 & 1 & 1 & 0 & 0\end{array}$ & $\begin{array}{lllllllll}0 & 0 & 0 & 0 & 0 & 1 & 1 & 1\end{array}$ & $\begin{array}{llllllll}1 & 0 & 1 & 0 & 1 & 0 & 0 & 1\end{array}$ & $\begin{array}{llllllll}1 & 0 & 1 & 0 & 1 & 0 & 1 & 1\end{array}$ & $\begin{array}{lllllllll}0 & 0 & 0 & 0 & 0 & 0 & 1 & 0\end{array}$ \\
\hline & $\begin{array}{lllllllll}0 & 1 & 0 & 1 & 0 & 1 & 0 & 0\end{array}$ & $\begin{array}{llllllllll}0 & 0 & 0 & 0 & 0 & 0 & 1 & 0 \\
0 & 0 & 0 & 0 & 1 & 1 & 0 & 0\end{array}$ & $\begin{array}{llllllll}1 & 0 & 1 & 1 & 0 & 1 & 0 & 0\end{array}$ & $\begin{array}{lllllll}10 & 1 & 1 & 0 & 0 & 0 & 0\end{array}$ & $\begin{array}{lllllllll}0 & 0 & 0 & 0 & 0 & 1 & 0 & 0\end{array}$ \\
\hline $\begin{array}{llllllllll}0 & 1 & 0 & 1 & 1 & 0 & 0 & 1 \\
0 & 1 & 1 & 0 & 0 & 0 & 1 & 1\end{array}$ & $\begin{array}{lllllllll}0 & 1 & 0 & 1 & 0 & 1 & 0 & 1 \\
0 & 1 & 1 & 1 & 0 & 0 & 0\end{array}$ & $\begin{array}{llllllll}0 & 0 & 0 & 1 & 0 & 0 & 0 & 0\end{array}$ & $\begin{array}{lllllllll}1 & 0 & 1 & 1 & 1 & 1 & 0 & 1\end{array}$ & $\begin{array}{lllllll}10 & 1 & 1 & 1 & 0 & 1 & 0\end{array}$ & $\begin{array}{lllllllll}0 & 0 & 0 & 0 & 0 & 1 & 1 & 1\end{array}$ \\
\hline $\begin{array}{lllllllll} & 0 & 1 & 1 & 0 & 0 & 1 & 0 & 1\end{array}$ & $\begin{array}{lllllllll}0 & 1 & 1 & 1 & 0 & 1 & 0 & 1\end{array}$ & $\begin{array}{lllllllll}0 & 0 & 0 & 1 & 0 & 0 & 0 & 0\end{array}$ & $\begin{array}{llllllll}1 & 1 & 0 & 0 & 0 & 1 & 1 & 0\end{array}$ & $\begin{array}{llllllll}1 & 1 & 0 & 0 & 0 & 1 & 0 & 0\end{array}$ & $\begin{array}{lllllllll}0 & 0 & 0 & 0 & 0 & 0 & 1 & 0\end{array}$ \\
\hline $\begin{array}{llllllll}0 & 1 & 1 & 0 & 1 & 0 & 1 & 0\end{array}$ & 0111111111 & $\begin{array}{lllllllll}0 & 0 & 0 & 1 & 0 & 1 & 0 & 1\end{array}$ & $\begin{array}{llllllll}1 & 1 & 0 & 0 & 1 & 0 & 0 & 1\end{array}$ & $\begin{array}{llllllll}1 & 1 & 0 & 0 & 1 & 0 & 0 & 0\end{array}$ & $\begin{array}{llllllll}0 & 0 & 0 & 0 & 0 & 0 & 0 & 1\end{array}$ \\
\hline $\begin{array}{lllllllll}0 & 1 & 1 & 0 & 1 & 1 & 0 & 0\end{array}$ & 010111111111 & $\begin{array}{lllllllll}0 & 0 & 0 & 1 & 0 & 0 & 1 & 1\end{array}$ & $\begin{array}{lllllllll}1 & 1 & 0 & 1 & 0 & 0 & 1 & 0\end{array}$ & $\begin{array}{llllllll}1 & 1 & 0 & 1 & 0 & 1 & 0 & 1\end{array}$ & $\begin{array}{lllllllll}0 & 0 & 0 & 0 & 0 & 1 & 1 & 1\end{array}$ \\
\hline $\begin{array}{lllllllll}0 & 1 & 1 & 1 & 1 & 0 & 0 & 0\end{array}$ & $\begin{array}{lllllllll}0 & 0 & 0 & 0 & 1 & 1 & 1 & 1\end{array}$ & $\begin{array}{lllllllll}0 & 1 & 1 & 1 & 0 & 1 & 1 & 1\end{array}$ & $\begin{array}{lllllllll}1 & 1 & 0 & 1 & 1 & 0 & 1 & 1\end{array}$ & $\begin{array}{lllllllllllll}1 & 1 & 0 & 1 & 1 & 1 & 0 & 0\end{array}$ & $\begin{array}{lllllllll}0 & 0 & 0 & 0 & 0 & 1 & 1 & 1\end{array}$ \\
\hline $\begin{array}{llllllllll}0 & 1 & 1 & 1 & 1 & 1 & 1 & 0\end{array}$ & $\begin{array}{llllllll}0 & 1 & 1 & 1 & 1 & 1 & 1 & 1\end{array}$ & $\begin{array}{llllllll}0 & 0 & 0 & 0 & 0 & 0 & 0 & 1\end{array}$ & $\begin{array}{lllllllll}1 & 1 & 1 & 0 & 0 & 0 & 0 & 1\end{array}$ & $\begin{array}{llllllll}1 & 1 & 1 & 0 & 0 & 0 & 0 & 0\end{array}$ & $\begin{array}{llllllll}0 & 0 & 0 & 0 & 0 & 0 & 0 & 1\end{array}$ \\
\hline 1000000001 & 10000000000 & $\begin{array}{llllllll}0 & 0 & 0 & 0 & 0 & 0 & 0 & 1\end{array}$ & $\begin{array}{lllllllll}1 & 1 & 1 & 0 & 0 & 1 & 1 & 1\end{array}$ & $\begin{array}{lllllllll}1 & 1 & 1 & 0 & 1 & 1 & 1 & 1\end{array}$ & $\begin{array}{lllllllll}0 & 0 & 0 & 0 & 1 & 0 & 0 & 0\end{array}$ \\
\hline
\end{tabular}

$f_{12}$ is also the $n-1$ variable of the function except $x_{1}$, and its RM expansion coefficient vector $B_{2}$ is equal to the last $n / 2$ elements of $B$.

(3) In the second decomposition, $f_{11}=f_{21} \oplus x_{2} f_{22}$, $f_{12}=f_{23} \oplus x_{2} f_{24}$, the subfunctions $f_{21}, f_{22}, f_{23}$, and $f_{24}$ are the $n-2$ variables of functions except $x_{1}$ and $x_{2}$, and their RM expansion coefficient vectors are equal to the first $(n-1) / 2$ elements of $B_{1}$, the last $(n-1) / 2$ elements of $B_{1}$, the first $(n-1) / 2$ elements of $B_{2}$, and the last $(n-1) / 2$ elements of $B_{2}$, respectively.

(4) Repeat step 3, until the subfunctions are the threevariable functions.

(5) Judging all the three-variable subfunctions, if the function is a threshold function, it can directly be implemented by 1 UTLG; if the function belongs to the first and fourth categories of the three-variable nonthreshold functions, it can be implemented by 3 UTLGs [14]; if the function belongs to the second and third categories of the three-variable nonthreshold functions, looking up the decomposition results from Tables 2 and 3, it can be implemented by 2 UTLGs and 1 XOR2; if the function is $f=x_{1} \oplus x_{2} \oplus x_{3}$ or $f=\overline{x_{1} \oplus x_{2} \oplus x_{3}}$, it can be implemented by 1 UTLG and 2 XOR2s.

(6) The $x_{i+1} f_{(i+1) l}$ of each decomposition equation $f_{i j}=$ $f_{(i+1) k} \oplus x_{i+1} f_{(i+1) l}$ can be regarded as the bivariate function, which can be implemented by 1 UTLG. And the bivariate XOR operation of the decomposition equations can be implemented by 1 XOR 2 .

Example 1. Implement the four-variable function:

$$
\begin{aligned}
f= & \overline{x_{1}} \overline{x_{3}} \overline{x_{4}}+\overline{x_{2}} \overline{x_{3}} \overline{x_{4}}+\overline{x_{1}} x_{2} \overline{x_{3}}+\overline{x_{1}} x_{2} \overline{x_{4}}+x_{1} x_{2} x_{4} \\
& +\overline{x_{1}} \overline{x_{2}} x_{3} x_{4} .
\end{aligned}
$$

The RM expansion coefficient vector $B$ of $f$ is $B=$ $\left[\begin{array}{llllllllllllllll}1 & 1 & 1 & 0 & 0 & 1 & 1 & 1 & 0 & 0 & 0 & 1 & 1 & 1 & 0 & 0\end{array}\right]$, the $f$ can be decomposed as $f=f_{11} \oplus x_{1} f_{12}, f_{11}$ is a three-variable function, and its RM expansion coefficient vector $B_{1}$ is equal to the first 8 elements of $B$; that is, $B_{1}=\left[\begin{array}{llllllll}1 & 1 & 1 & 0 & 0 & 1 & 1 & 1\end{array}\right]$, $f_{11}=x_{2} \overline{x_{3}}+x_{2} \overline{x_{4}}+\overline{x_{3}} \overline{x_{4}}+\overline{x_{2}} x_{3} x_{4} \cdot f_{12}$ is a three-variable function, and its RM expansion coefficient vector $B_{2}$ is equal to the last 8 elements of $B$; that is, $B_{2}=\left[\begin{array}{llllllll}0 & 0 & 0 & 1 & 1 & 1 & 0 & 0\end{array}\right]$, $f_{12}=x_{2} \overline{x_{4}}+x_{3} x_{4}$. 


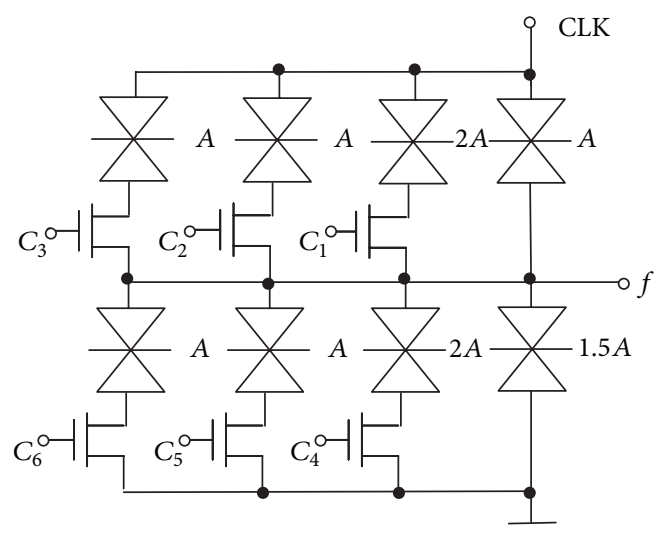

(a)

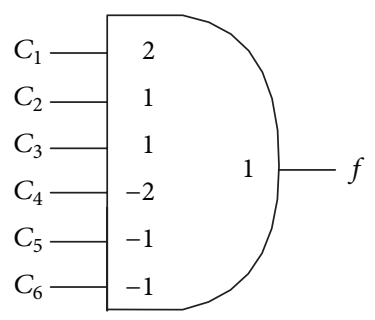

(b)

Figure 1: The universal threshold logic gate (UTLG), (a) schematic (b) symbol.

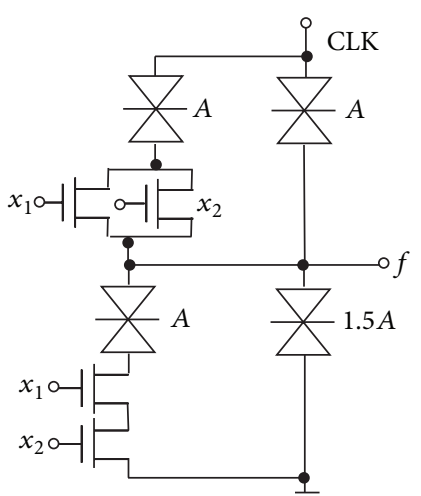

(a)

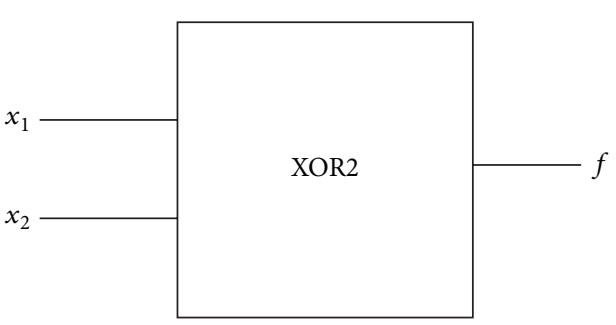

(b)

Figure 2: The bivariate XOR gate (XOR2), (a) schematic (b) symbol.

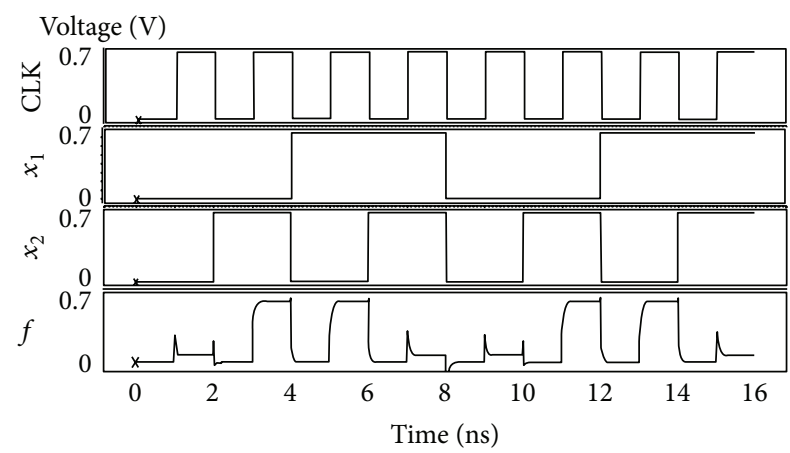

FIgURE 3: Transient waveforms of the proposed XOR2.

$f_{11}$ belongs to the second category of the three-variable nonthreshold functions; looking up Table 2, $f_{11}=f_{11}^{\prime} \oplus f_{12}^{\prime}$, $f_{11}^{\prime}=\left\langle x_{3}+x_{4}\right\rangle_{2}, f_{12}^{\prime}=\left\langle 2 x_{2}-x_{3}-x_{4}\right\rangle_{0} . f_{12}$ belongs to the fourth category of the three-variable nonthreshold functions, and it can be decomposed as [14] $f_{12}=f_{21}^{\prime}+f_{22}^{\prime}, f_{21}^{\prime}=x_{2} \overline{x_{4}}$, $f_{22}^{\prime}=x_{3} x_{4}$. Then $f$ can be implemented by 6 UTLGs and 2 XOR2s. Figure 4 shows the UTLG and XOR2 implementation of this function.
Example 2. Implement the five-variable function:

$$
\begin{aligned}
f= & x_{1} x_{2} \overline{x_{3}} \overline{x_{4}} x_{5}+x_{1} \overline{x_{2}} \overline{x_{3}} x_{4} \overline{x_{5}}+\overline{x_{1}} \overline{x_{3}} x_{4} x_{5} \\
& +\overline{x_{1}} x_{2} \overline{x_{3}} x_{4}+x_{1} x_{2} x_{3} x_{4}+\overline{x_{1}} x_{3} \overline{x_{4}} x_{5} \\
& +\overline{x_{2}} x_{3} \overline{x_{4}} \overline{x_{5}}+\overline{x_{1}} \overline{x_{2}} x_{3} .
\end{aligned}
$$

The RM expansion coefficient vector $B$ of $f$ is $B=$ $\left[\begin{array}{llllllllllllllllllllll}0 & 0 & 0 & 1 & 1 & 0 & 0 & 1 & 0 & 0 & 1 & 1 & 1 & 1 & 1 & 0 & 0 & 0 & 1 & 0 & 0 & 1\end{array}\right.$ $\left.\begin{array}{llllllllll}0 & 1 & 0 & 1 & 0 & 1 & 0 & 1 & 0 & 1\end{array}\right], f$ can be decomposed as $f=$ $f_{11} \oplus x_{1} f_{12}, f_{11}$ is a four-variable function, its RM expansion coefficient vector $B_{1}$ is equal to the first 16 elements of $B$, that is, $B_{1}=\left[\begin{array}{llllllllllllllll}0 & 0 & 0 & 1 & 1 & 0 & 0 & 1 & 0 & 0 & 1 & 1 & 1 & 1 & 1 & 0\end{array}\right], f_{12}$ is a four-variable function, and its RM expansion coefficient vector $B_{2}$ is equal to the last 16 elements of $B$, that is, $B_{2}=\left[\begin{array}{llllllllllllllll}0 & 0 & 1 & 0 & 0 & 1 & 0 & 1 & 0 & 1 & 0 & 1 & 0 & 1 & 0 & 1\end{array}\right]$.

Continue to decompose $f_{11} \cdot f_{11}=f_{21} \oplus x_{2} f_{22}, f_{21}$ is a three-variable function, its RM expansion coefficient vector $B_{3}$ is equal to the first 8 elements of $B_{1}$, that is, $B_{3}=$ $\left[\begin{array}{llllllll}0 & 0 & 0 & 1 & 1 & 0 & 0 & 1\end{array}\right], f_{22}$ is also a three-variable function, and its RM expansion coefficient vector $B_{4}$ is equal to the last 8 elements of $B_{1}$, that is, $B_{4}=\left[\begin{array}{llllllll}0 & 0 & 1 & 1 & 1 & 1 & 1 & 0\end{array}\right]$.

Continue to decompose $f_{12} \cdot f_{12}=f_{23} \oplus x_{2} f_{24}, f_{23}$ is a three-variable function, its RM expansion coefficient vector $B_{5}$ is equal to the first 8 elements of $B_{2}$, that is, 


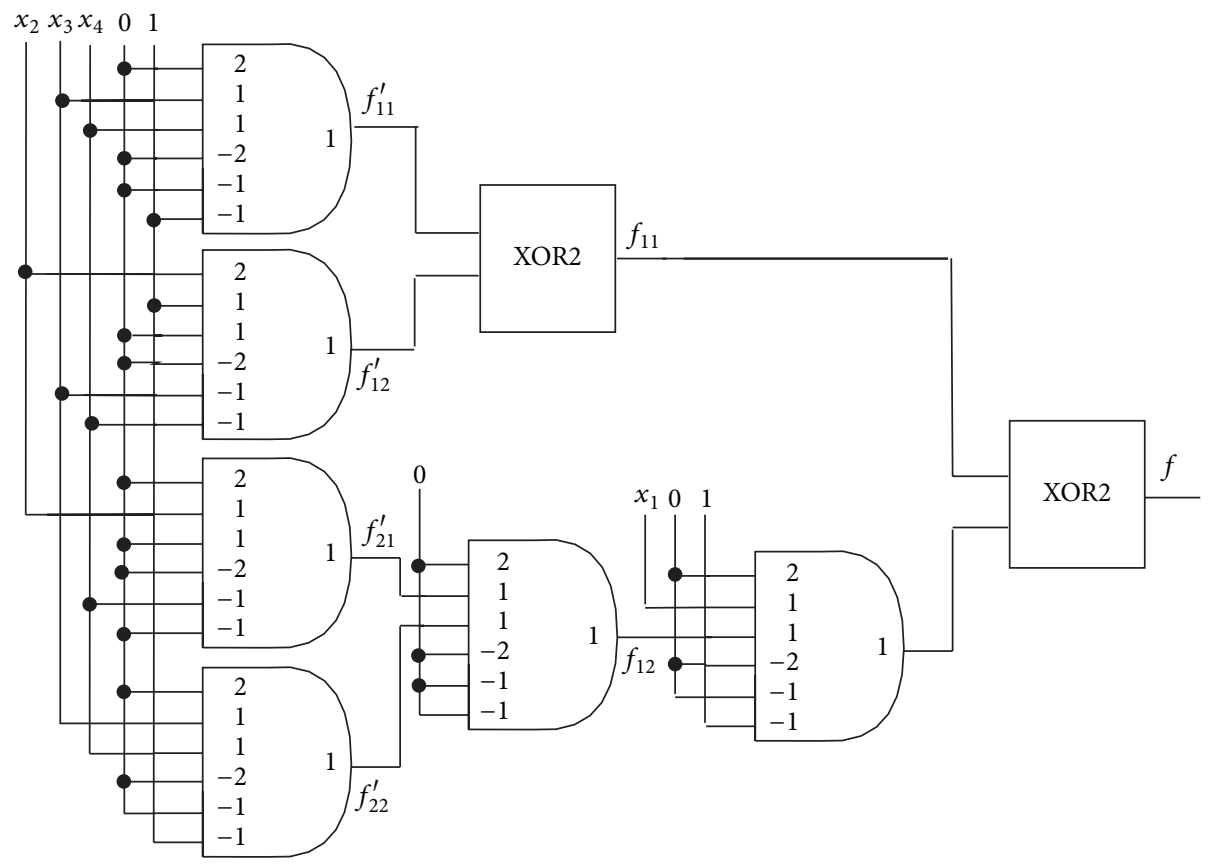

FIgURE 4: UTLG and XOR2 implementation of Example 1.

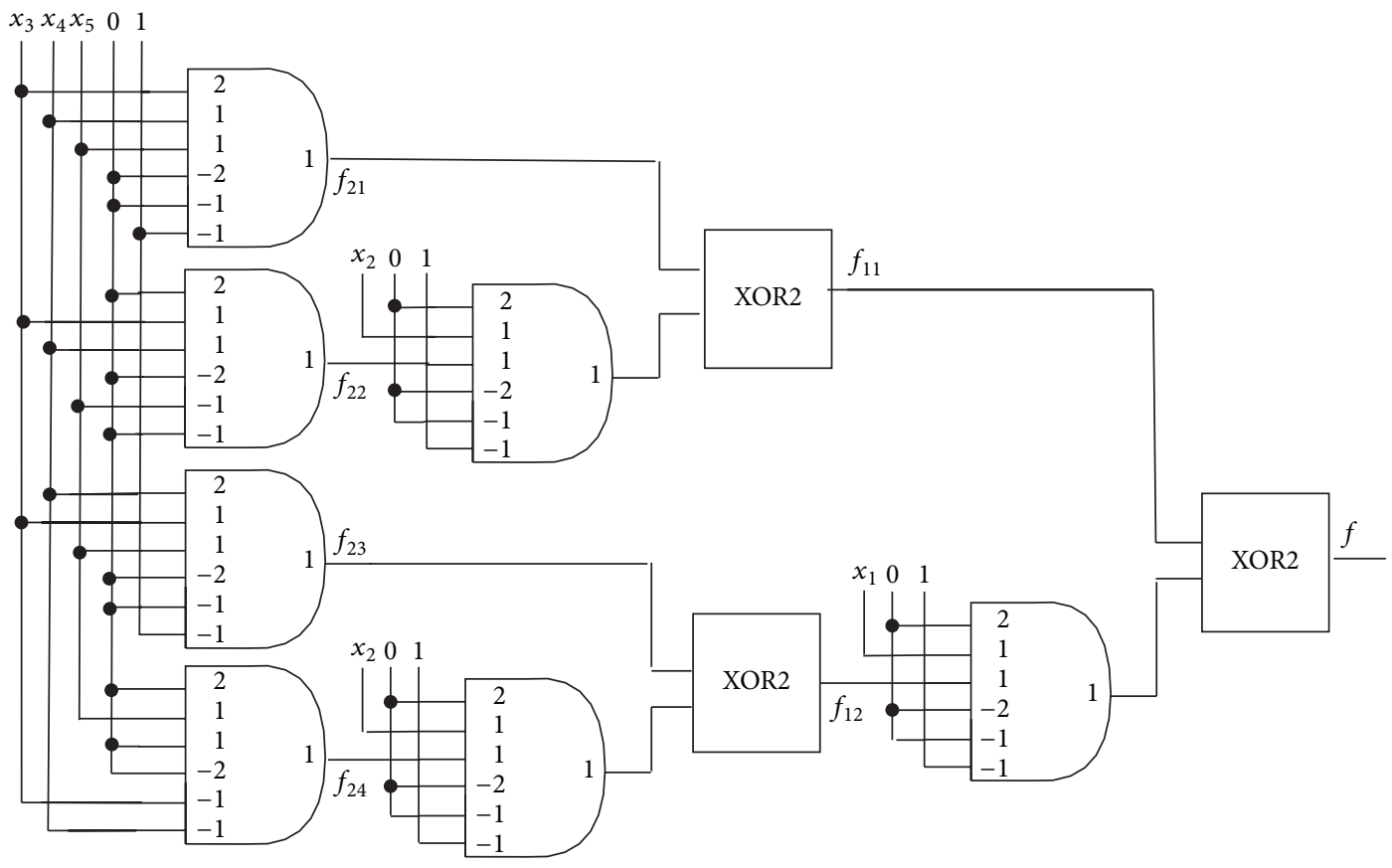

FIgURE 5: UTLG and XOR2 implementation of Example 2.

$B_{5}=\left[\begin{array}{llllllll}0 & 0 & 1 & 0 & 0 & 1 & 0 & 1\end{array}\right], f_{24}$ is a three-variable function, and its RM expansion coefficient vector $B_{6}$ is equal to the last 8 elements of $B_{2}$, that is, $B_{6}=\left[\begin{array}{llllllll}0 & 1 & 0 & 1 & 0 & 1 & 0 & 1\end{array}\right]$.

$f_{21}, f_{22}, f_{23}$, and $f_{24}$ are the three-variable threshold functions, and $f$ can be implemented by 7 UTLGs and 3 XOR2s. Figure 5 shows the UTLG and XOR2 implementation of this function.
The proposed synthesis algorithm of the $n$-variable function based on UTLG can decompose the arbitrary $n$ variable function into three-variable functions, and all the three-variable nonthreshold functions are divided into four categories, and two categories of them can be implemented by UTLG and XOR2. Thus, arbitrary $n$-variable functions can be implemented by UTLG and XOR2, and if the given 
function is decomposed into the threshold functions, the circuit structure will be simple.

Up to now, there is no algorithm which can implement arbitrary $n$-variable function by UTLG. Reference [13] just proposed the algorithm which can implement arbitrary three-variable function. So, we provided new logic units UTLG and XOR2 and a new algorithm to implement RTDbased arbitrary $n$-variable function.

\section{Conclusion}

In this paper, the 152 three-variable nonthreshold functions are divided into four categories, and a new decomposition algorithm of the three-variable nonthreshold functions is proposed. If the function belongs to the second and third categories of the three-variable nonthreshold functions, it can be implemented by 2 UTLGs and 1 XOR2, and it simplifies the implemented circuit structure. Based on the Reed-Muller algebraic system, the arbitrary $n$-variable function can be decomposed into three-variable functions, and the function synthesis algorithm for the $n$-variable function which can be implemented by UTLG and XOR2 is proposed; that is, the arbitrary $n$-variable function can be implemented by UTLG and XOR2, and if the given function is decomposed into three-variable threshold functions, the circuit structure will be much simpler. The proposed logic units and algorithm present a new method to implement the arbitrary $n$-variable function by RTD-based devices.

\section{Conflict of Interests}

The authors declare that there is no conflict of interests regarding the publication of this paper.

\section{Acknowledgments}

This work was supported by the National Natural Science Foundation of China under Grant nos. 61271124 and 61471314 and Zhejiang Provincial Natural Science Foundation of China under Grant nos. LY13F010001 and LY15F010011.

\section{References}

[1] N. Muramatsu, H. Okazaki, and T. Waho, "A novel oscillation circuit using a resonant-tunneling diode," in Proceedings of the IEEE International Symposium on Circuits and Systems (ISCAS '05), pp. 2341-2344, May 2005.

[2] P. Mazumder, S. Kulkarni, M. Bhattacharya, J. P. Sun, and G. I. Haddad, "Digital circuit applications of resonant tunneling devices," Proceedings of the IEEE, vol. 86, no. 4, pp. 664-686, 1998.

[3] X. Chen and S. L. Hurst, "A consideration of the minimum number of input terminals on universal logic gates and their realization," International Journal of Electronics, vol. 50, no. 1, pp. 1-13, 1981.

[4] V. Beiu, J. M. Quintana, and M. J. Avedillo, "implementations of threshold logic - a comprehensive survey," IEEE Transactions on Neural Networks, vol. 14, no. 5, pp. 1217-1243, 2003.
[5] Y. X. Zheng and C. Huang, "Complete logic functionality of reconfigurable RTD circuit elements," IEEE Transactions on Nanotechnology, vol. 8, no. 5, pp. 631-642, 2009.

[6] S. M. Mirhoseini, M. J. Sharifi, and D. Bahrepour, "New RTDbased general threshold gate topologies and application to three-input XOR logic gates," Journal of Electrical and Computer Engineering, vol. 35, no. 1, Article ID 463925, pp. 1-4, 2010.

[7] K. K. Likharev, "Hybrid CMOS/nanoelectronic circuits: opportunities and challenges," Journal of Nanoelectronics and Optoelectronics, vol. 3, no. 3, pp. 203-230, 2008.

[8] J. Lee, S. Choi, and K. Yang, "A new low-power RTD-based 4:1 multiplexer IC using an InP RTD/HBT MMIC technoligy," in Proceedings of the 22nd International Conference on Indium Phosphide and Related Materials, pp. 1-3, Kagawa, Japan, June 2010.

[9] S. Kolłodziński and E. Hrynkiewicz, "An utilisation of boolean differential calculus in variables partition calculation for decomposition of logic functions," in Proceedings of the IEEE Symposium on Design and Diagnostics of Electronic Circuits \& Systems (DDECS '09), pp. 34-37, April 2009.

[10] E. Hrynkiewicz and S. Kołodziński, "Non-disjoint decomposition of logic functions in Reed-Müller spectral domain," in Proceedings of the 13th IEEE International Symposium on Design and Diagnostics of Electronic Circuits and Systems (DDECS '10), pp. 293-296, IEEE, Vienna, Austria, April 2010.

[11] E. Hrynkiewicz and S. Kołodziński, "An Ashenhurst disjoint and non-disjoint decomposition of logic functions in ReedMuller spectral domain," in Proceedings of the 17th International Conference on Mixed Design of Integrated Circuits and Systems (MIXDES '10), pp. 200-204, June 2010.

[12] F. Yu, L. F. Wang, R. H. Tan, and H. Jin, "An improved functional decomposition method based on FAST and the method of removal and operation," in Proceedings of the International Conference on System Science and Engineering (ICSSE '12), pp. 487-492, Dalian, China, July 2012.

[13] Y. Wei and J. Z. Shen, "Novel universal threshold logic gate based on RTD and its application," Microelectronics Journal, vol. 42, no. 6, pp. 851-854, 2011.

[14] R. Zhang, P. Gupta, L. Zhong, and N. K. Jha, “Threshold network synthesis and optimization and its application to nanotechnologies," IEEE Transactions on Computer-Aided Design of Integrated Circuits and Systems, vol. 24, no. 1, pp. 107-118, 2005.

[15] S. Muroga, Threshold Logic and Its Application, John Wiley \& Sons, New York, NY, USA, 1971.

[16] S. L. Hurst, J. C. Muzio, and D. M. Miller, Spectral Techniques in Digital Logic, Academic Press, London, UK, 1985.

[17] K. L. Kodandapani and R. V. Setlur, "A note on minimal Reed-Muller canonical forms of switching functions," IEEE Transactions on Computers, vol. 26, no. 3, pp. 310-313, 1977.

[18] K. J. Chen and K. Maezawa, "Monostable-bistable transition logic elements (MOBILES) based on monolithic integration of resonant tunneling diodes and FETs," Japanese Journal of Applied Physics, vol. 34, no. 2, pp. 1199-1203, 1995. 


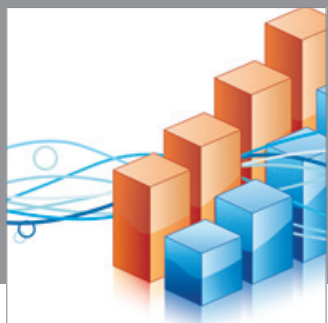

Advances in

Operations Research

mansans

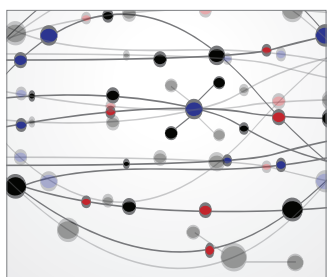

The Scientific World Journal
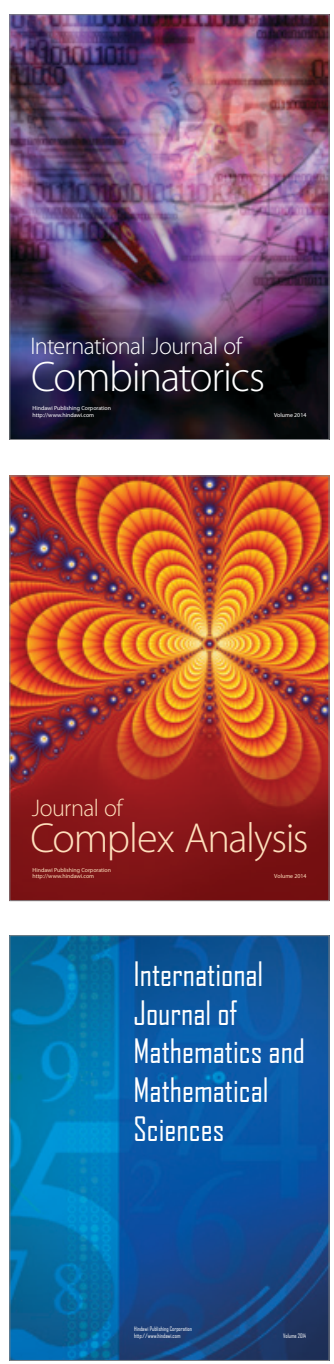
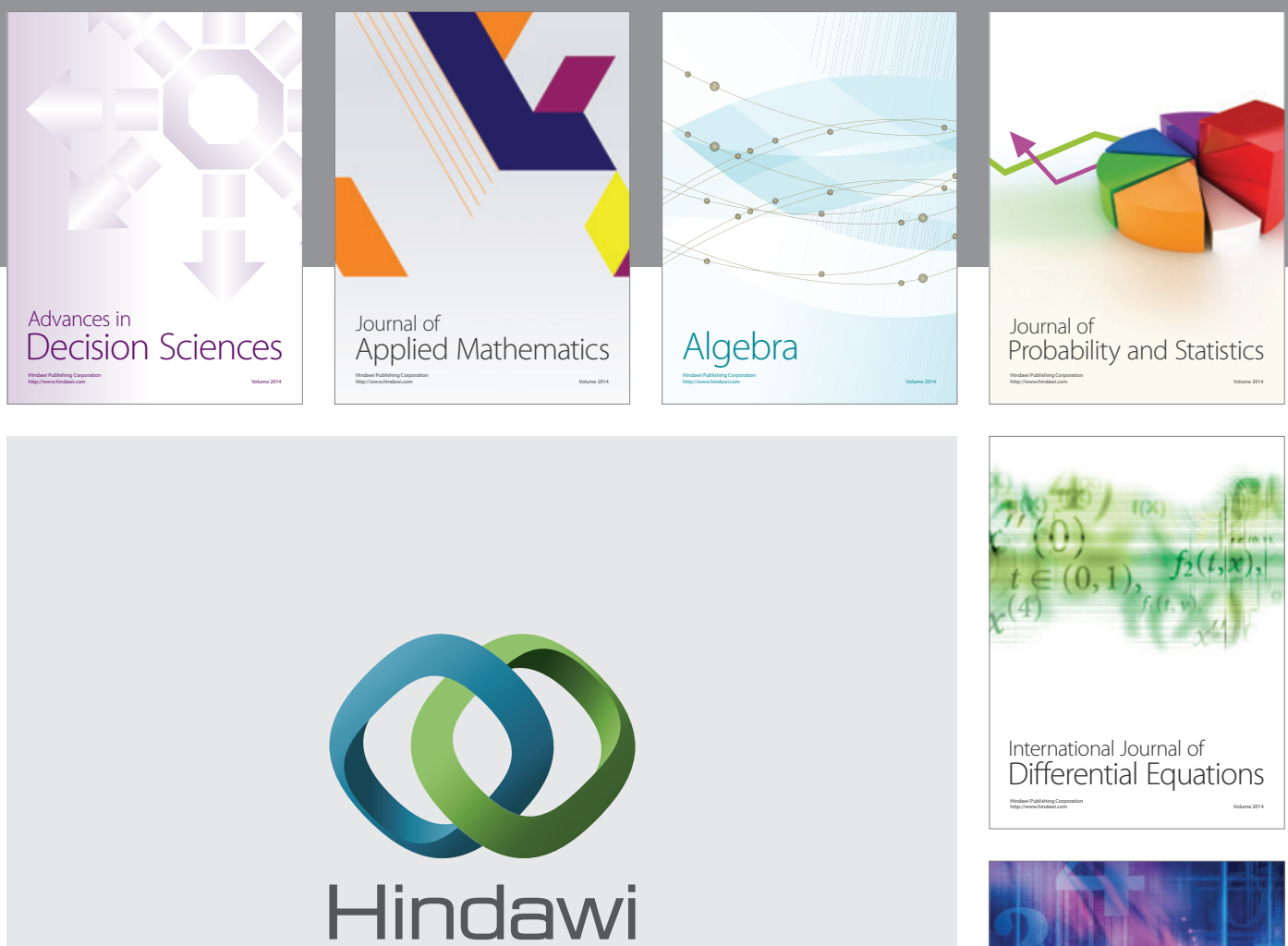

Submit your manuscripts at http://www.hindawi.com
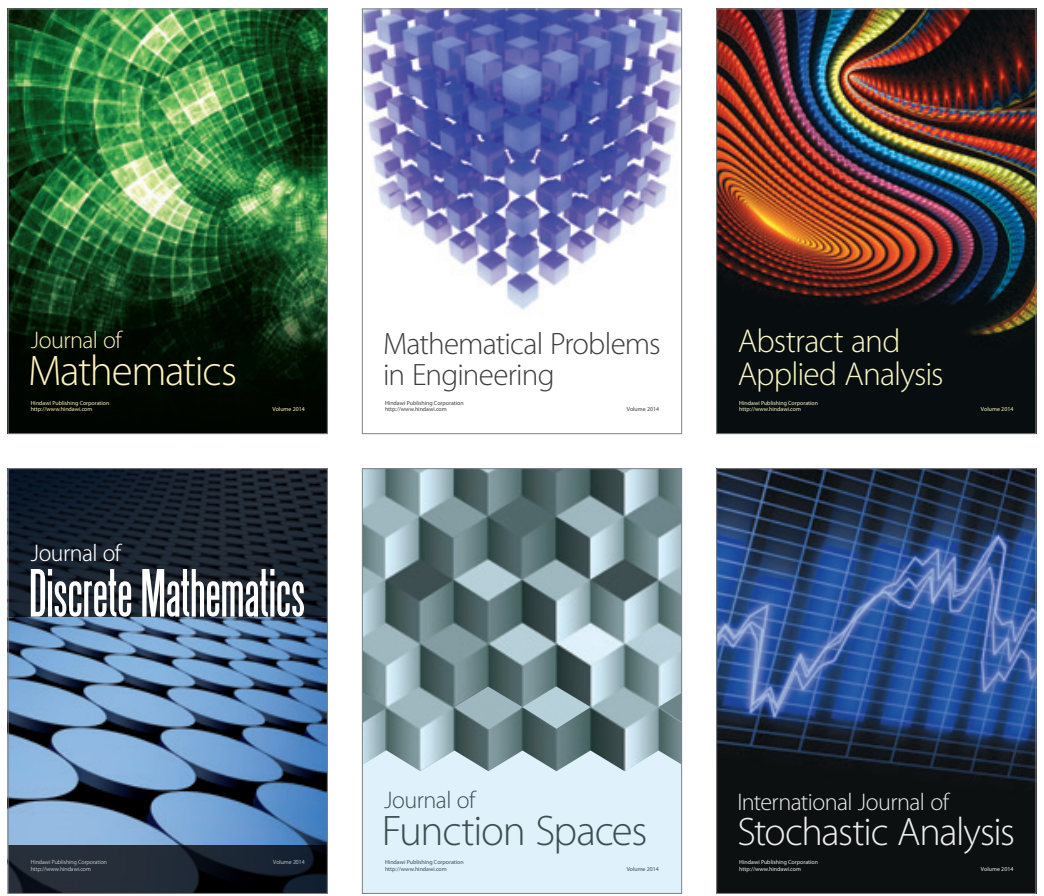

Journal of

Function Spaces

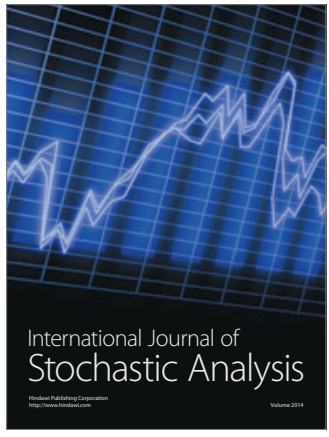

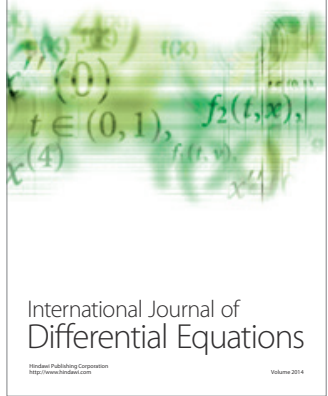
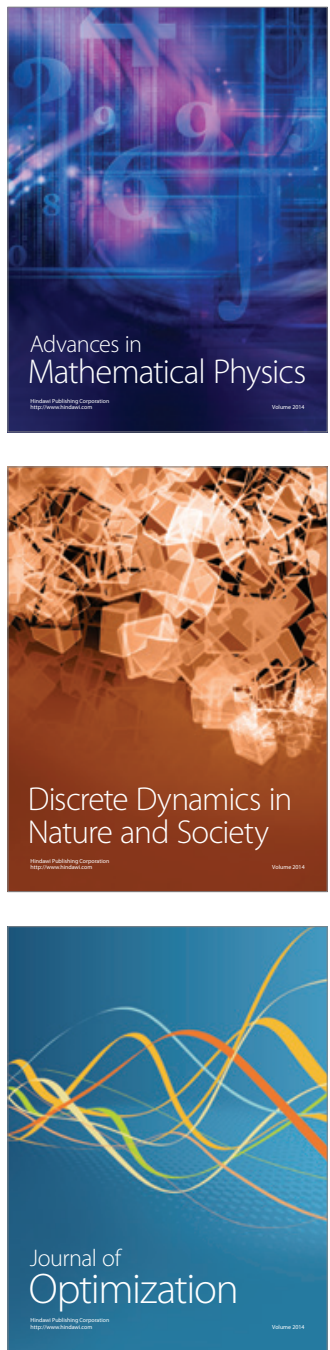